\title{
How vestibular stimulation interacts with illusory hand ownership
}

\author{
Christophe Lopez ${ }^{\mathrm{a}, *}$, Bigna Lenggenhager ${ }^{\mathrm{a}}$, Olaf Blanke ${ }^{\mathrm{a}, \mathrm{b}}$ \\ ${ }^{a}$ Laboratory of Cognitive Neuroscience, Brain-Mind Institute, Ecole Polytechnique Fédérale de Lausanne, Station 19 , 1015 Lausanne, Switzerland \\ ${ }^{\mathrm{b}}$ Department of Neurology, University Hospital, Geneva, Switzerland
}

\section{A R T I C L E I N F O}

\section{Article history:}

Received 2 September 2009

Available online 4 January 2010

\section{Keywords:}

Vestibular system

Touch

Multisensory integration

Visual capture

Somatoparaphrenia

Consciousness

Galvanic vestibular stimulation

Bodily illusions

Rubber hand illusion

\begin{abstract}
A B S T R A C T
Artificial stimulation of the peripheral vestibular system has been shown to improve ownership of body parts in neurological patients, suggesting vestibular contributions to bodily self-consciousness. Here, we investigated whether galvanic vestibular stimulation (GVS) interferes with the mechanisms underlying ownership, touch, and the localization of one's own hand in healthy participants by using the "rubber hand illusion" paradigm. Our results show that left anodal GVS increases illusory ownership of the fake hand and illusory location of touch. We propose that these changes are due to vestibular interference with spatial and/or temporal mechanisms of visual-tactile integration leading to an enhancement of visual capture. As only left anodal GVS lead to such changes, and based on neurological data on body part ownership, we suggest that this vestibular interference is mediated by the right temporo-parietal junction and the posterior insula.
\end{abstract}

(c) 2009 Elsevier Inc. All rights reserved.

\section{Introduction}

A long-standing and central theme in philosophy, human self-consciousness, has in recent years also become an increasingly prominent issue in cognitive neuroscience (Gallagher, 2000). Whereas earlier research focused mainly on higher-level aspects such as memory, personality, or language, recent studies have started to investigate more basic, i.e. non-conceptual, aspects of self-consciousness. This growing amount of neuroscientific data and philosophical theories suggest that fundamental aspects of self-consciousness (bodily self-consciousness) may be related to brain mechanisms encoding the different multisensory and sensorimotor states of the body (Berlucchi \& Aglioti, 1997, 2009; Blanke \& Metzinger, 2009; Botvinick, 2004; Damasio, 2000; Jeannerod, 2006; Vogeley et al., 2004).

Ownership for one's body and its parts as well as their localization in space have been proposed to constitute crucial aspects of bodily self-consciousness (de Vignemont, 2007; Gallagher, 2000; Tsakiris, Schutz-Bosbach, \& Gallagher, 2007). Over the last years an increasing number of empirical data on the neural underpinnings of body ownership and body localization have pointed to the importance of multisensory integration of visual, tactile and proprioceptive signals. These data suggest that differentiating one's own body parts from external objects and self-attributing these as belonging to one's own body is based on matching multiple sensory signals in time and space (Botvinick, 2004; Botvinick \& Cohen, 1998; Ehrsson, Holmes, \& Passingham, 2005). The synchronous experience of the sight of an object touching our arm and a tactile sensation originating from that arm may lead us for example to self-attribute this arm as ours. Similarly, the ability to localize one's own body parts in space depends on the congruence between the seen position of one's own arm and the position of one's arm coded by the proprioceptive system.

\footnotetext{
* Corresponding author. Fax: +4102169317 70.

E-mail addresses: christophe.lopez@epfl.ch, christophe.g.lopez@gmail.com (C. Lopez).
} 
The integration of these multisensory bodily signals into one coherent representation is thus necessary for normal bodily self-consciousness. A failure of such integration may evoke abnormal hand ownership and hand localization, as it is sometimes observed in epileptic, vascular stroke, or deafferented patients (Fourneret, Paillard, Lamarre, Cole, \& Jeannerod, 2002; Hécaen \& de Ajuriaguerra, 1952; Vallar \& Ronchi, 2009). For example, in brain-damaged patients suffering from somatoparaphrenia, ownership for body parts is severely impaired (Aglioti, Smania, Manfredi, \& Berlucchi, 1996; Bisiach, Rusconi, \& Vallar, 1991; Bottini, Bisiach, Sterzi, \& Vallar, 2002; Schiff \& Pulver, 1999; Vallar \& Ronchi, 2009). These patients do not self-attribute correctly their own body parts (mostly the contralesional hand) or self-attribute someone else's hand as belonging to them. This condition is mainly observed after damage to the right parietotemporal cortex, which has been shown to be crucial for the integration of visual, tactile and proprioceptive signals (Bense, Stephan, Yousry, Brandt, \& Dieterich, 2001; Brandt \& Dieterich, 1999; Guldin \& Grüsser, 1998). Studies performed in healthy subjects confirmed the importance of multisensory integration for bodily self-consciousness by showing that experimentally induced multisensory conflicts may temporarily disturb body ownership as well as self-localization. The most commonly used paradigm to manipulate ownership and localization of body parts is the so-called "rubber hand illusion" (Armel \& Ramachandran, 2003; Botvinick, 2004; Botvinick \& Cohen, 1998; Costantini \& Haggard, 2007; Ehrsson, Wiech, Weiskopf, Dolan, \& Passingham, 2007; Ehrsson et al., 2005; Kammers, de Vignemont, Verhagen, \& Dijkerman, 2009; Lloyd, 2007; Longo, Schuur, Kammers, Tsakiris, \& Haggard, 2008; Moseley et al., 2008; Pavani, Spence, \& Driver, 2000; Schutz-Bosbach, Tausche, \& Weiss, 2009; Tsakiris \& Haggard, 2005). The rubber hand illusion is usually evoked by synchronously stroking the participant's hidden hand and a seen rubber hand placed in front of the participant. This manipulation creates illusory ownership for the rubber hand (Botvinick \& Cohen, 1998). In addition, due to the spatio-temporal matching between the touch seen on the rubber hand and the touch felt on the real hand, subjects report the touch to originate from where it is seen (thus on the rubber hand). Finally, participants mislocalize their own hand towards the rubber hand, often called "proprioceptive drift" (Botvinick \& Cohen, 1998).

What other sensory signals may be important for bodily self-consciousness? Vestibular signals code for a large range of angular and linear accelerations, including gravitational acceleration, and they therefore play a prominent role in determining one's own body motion and orientation in space (Day \& Fitzpatrick, 2005). Vestibular signals also contribute to spatial navigation and to the distinction between self-motion and non-self motion (Dichgans \& Brandt, 1978). For these tasks, the vestibular system does not operate in isolation but strongly interacts with the somatosensory and visual systems (Angelaki \& Cullen, 2008). Despite the importance of vestibular signals for coding one's own body location with respect to the environment, little is known about its contribution to the mechanisms of self-consciousness (see recent reviews in Lenggenhager, Smith, \& Blanke, 2006; Lopez \& Blanke, 2007; Lopez, Halje, \& Blanke, 2008; Miller \& Ngo, 2007). Yet, several neurological observations suggest a vestibular contribution to bodily self-consciousness (Bisiach et al., 1991; Blanke, Landis, Spinelli, \& Seeck, 2004; Blanke, Ortigue, Landis, \& Seeck, 2002; Hécaen \& de Ajuriaguerra, 1952; Ramachandran \& McGeoch, 2007; Rode et al., 1992; Schiff \& Pulver, 1999; Vallar, Bottini, Rusconi, \& Sterzi, 1993). Of pertinence for the present study, Bisiach et al. (1991) described a patient suffering from somatoparaphrenia (this patient misidentified her left arm as belonging to her mother), whose abnormal ownership for her left arm was transiently normalized by caloric vestibular stimulation. In the same line, artificial stimulation of the peripheral vestibular system may modify the experience of phantom limbs in amputees and paraplegic patients (André, Martinet, Paysant, Beis, \& Le Chapelain, 2001; Le Chapelain, Beis, Paysant, \& Andre, 2001), strengthening the idea of an influence of vestibular processing on body part illusions. The contribution of vestibular cues to hand localization has not been specifically investigated in neurological patients, however, in healthy subjects, vestibular stimulation has been shown to induce errors during pointing movements to one's own hand (Bresciani et al., 2002; Mars, Archambault, \& Feldman, 2003) and reproduction of arm positions (Knox, Coppieters, \& Hodges, 2006).

To the best of our knowledge, so far there has been no attempt to elucidate, by an experimental approach in healthy participants, the nature of the contribution of vestibular cues to illusory touch, hand ownership, and hand localization. In the present study, we investigated whether galvanic vestibular stimulation (GVS) interferes with the mechanisms underlying ownership and localization of body parts in healthy participants using the "rubber hand illusion" paradigm (Botvinick \& Cohen, 1998; Tsakiris \& Haggard, 2005). We employed GVS because it has been shown to be a powerful method to experimentally manipulate the firing rate of the peripheral vestibular apparatus, evoking illusory body motion in a predictable way (Fitzpatrick \& Day, 2004; Fitzpatrick, Marsden, Lord, \& Day, 2002; Lenggenhager, Lopez, \& Blanke, 2008; Mars, Vercher, \& Popov, 2005). Although previous clinical studies have used caloric vestibular stimulation to interfere with bodily experience - including somatoparaphrenia - in neurological patients, GVS was chosen here because the intensity and duration of the electrical stimulation can be set very precisely, whereas for caloric vestibular stimulation it can be difficult to precisely control the strength and duration of the irrigation. Here, we measured illusory ownership for a left rubber hand as well as the proprioceptive drift commonly induced by the rubber hand illusion manipulation during right or left anodal GVS, during right or left sham stimulations (on the neck), and during a baseline without any galvanic stimulation. First, based on clinical data in patients suffering from somatoparaphrenia (Bisiach et al., 1991; Rode et al., 1992; Schiff \& Pulver, 1999), we hypothesized that GVS, but not sham stimulation, would influence ownership for the rubber hand during the illusion, thus during synchronous stroking. Second, we predicted differences between right and left anodal GVS. We hypothesized that the illusory ownership for the left hand would be specifically more influenced by the 'left anodal-right cathodal' GVS configuration (that is activating the right labyrinth). This is, because vestibular stimulations that activate the right labyrinth (or inhibit the left labyrinth) activate predominantly the right 
cerebral hemisphere (Bisiach et al., 1991; Rode et al., 1992, 2002; Vallar et al., 1993) in which the tested real and fake hands are represented. Third, based on the results from Bresciani et al. (2002) and Knox et al. (2006) we predicted that GVS would lead to a mislocalization of the participant's hand, possibly interfering with the proprioceptive drift evoked during the rubber hand illusion.

\section{Materials and methods}

\subsection{Participants}

The data were obtained from 16 healthy paid volunteers ( 9 men, ages: $18-30$ years, mean \pm SD: $24 \pm 4$ years). All of them had normal or corrected-to-normal vision and none reported a history of neuro-otological disorder. All participants were right-handed. Informed consent was obtained from all participants prior to their inclusion in the study. The study protocol was approved by the local ethics research committee at the University of Lausanne and was performed in accordance with the ethical standards laid down in the Declaration of Helsinki.

\subsection{Galvanic vestibular stimulation}

Galvanic vestibular stimulation (GVS) was applied in the present investigation because it is a well-controlled way of artificially stimulating the vestibular receptors (Fitzpatrick \& Day, 2004), whereas caloric vestibular stimulations, that has been used in clinical studies to alleviate bodily symptoms in neurological patients, may provide stronger vestibular disturbance, but possibly in a less controlled way. An important advantage of GVS is that the intensity of the electrical stimulation can be set very precisely and adapted for each single participant (Lenggenhager et al., 2008). By contrast, for caloric vestibular stimulations, the ears are usually irrigated with cold or warm water with fixed temperature, and the intensity of stimulation is not individually adapted to the participant. For caloric vestibular stimulation, it is also difficult to precisely control the volume of water going into the external ear canal and thus to precisely control the parameters of the stimulation. Second, the duration of GVS can be selected very precisely (with ms-accuracy), whereas it is impossible to stop the caloric stimulation with high temporal accuracy, because the water cannot be removed from the external ear canal that fast and the caloric effects may persist. Finally, we wanted to minimize the visual side effects of the artificial vestibular stimulation and caloric stimulation has been reported to evoke a strong nystagmus (Aw, Haslwanter, Fetter, \& Dichgans, 2000), visual illusions and hallucinations (Kolev, 1995; Yen Pik Sang, Jauregui-Renaud, Green, Bronstein, \& Gresty, 2006).

GVS was applied using a bipolar, binaural configuration. A stimulator (Model Grass S48, Astor-Med Inc., West Warwick, RI, USA) was used to deliver a square wave pulse through an isolation unit (Model Grass SIU5) and a constant current stimulus unit (Model Grass CCU1) to the electrodes. This setting provides a safe and constant current output with the anode on one side and the cathode on the other side (Fig. 1A). Electrodes consisted of carbon rubber electrodes $(4 \times 5 \mathrm{~cm})$ embedded in a sponge saturated with water. They were fixed to the participant's head using flexible Velcro strips. For the GVS, the electrodes were placed on the left and right mastoid processes. We also applied sham stimulation, evoking similar tactile stimulation but no vestibular stimulation (see Lenggenhager et al., 2008), which is comparable to the standard approach used in transcranial magnetic stimulation studies. For this, the electrodes were placed on the left and right side of the neck, about $5 \mathrm{~cm}$ below the GVS electrodes. According to our convention 'right GVS' or 'right sham' stimulation refers to right anodal stimulations, and 'left GVS' or 'left sham' stimulation refers to left anodal stimulations. Using such a binaural bipolar configuration, GVS is known to increase the firing rate in vestibular afferents on the cathodal side and to decrease the firing rate on the anodal side (Goldberg, Smith, \& Fernandez, 1984). This change in the firing rate has been associated with illusory movements of both own body and visual field (Fitzpatrick \& Day, 2004; Fitzpatrick et al., 2002; Lenggenhager et al., 2008; Mars et al., 2005; Zink, Bucher, Weiss, Brandt, \& Dieterich, 1998).

The individual amplitude of the electrical stimulation was determined by progressively increasing the current amplitude separately for right and left GVS, as well as for right and left sham stimulations (as in Lenggenhager et al., 2008). For GVS, the current amplitude was first progressively increased until the participants (who were naïve to the effects of GVS) reported vestibular sensations. Then, accounting for the fact that higher current amplitudes lead to stronger vestibular effects (given that the vestibular threshold is reached), the amplitude was further increased to maximize the vestibular effects until participants reported the stimulation to be uncomfortable. If too uncomfortable, the amplitude of the stimulation was decreased. Thus, the stimulation intensity used was a suprathreshold current that was chosen at a level that was a compromise between inducing as strong vestibular illusions as possible while maintaining tolerable sensations of the stimulated skin. The mean current amplitude did not differ between left GVS (mean: $1.7 \pm 0.3 \mathrm{~mA}$ ) and right GVS (1.6 $\pm 0.2 \mathrm{~mA}$; Student's $t$-test: $P=.36)$. For the sham stimulations, the stimulation amplitude was fixed so that subjects reported approximately the same intensity of tactile or heat sensation on the skin as during GVS. The mean current amplitude was similar for left $(1.5 \pm 0.6 \mathrm{~mA})$ and right sham stimulations $(1.5 \pm 0.5 \mathrm{~mA}$; Student's $t$-test: $P=.72)$. There was also no difference between the mean current amplitude for GVS and sham stimulations (Student's $t$-test: anode left, $P=.18$; anode right, $P=.13$ ). 
A

GVS left
(anode left)

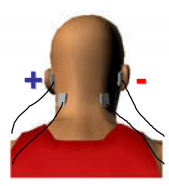

GVS right

(anode right)

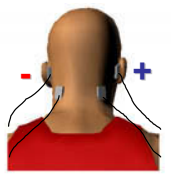

Sham left

(anode left)
Sham right

(anode right)
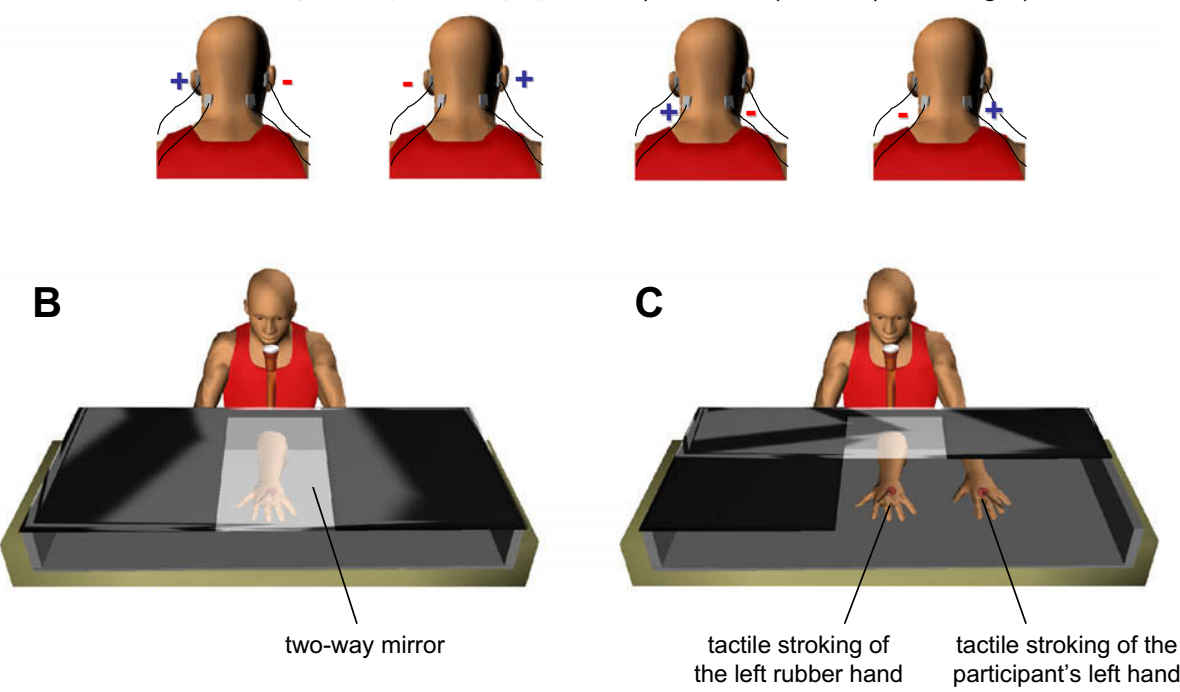

D
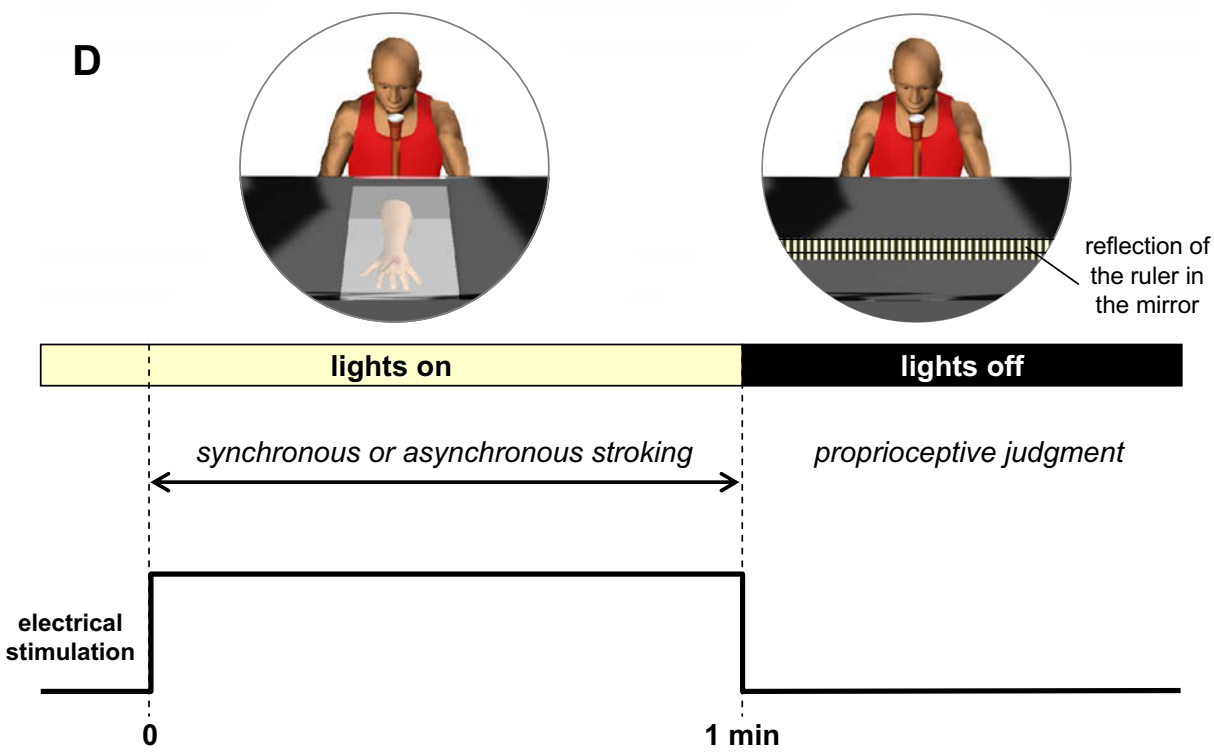

lights off

proprioceptive judgment

the left rubber hand

tactile stroking of the
participant's left hand

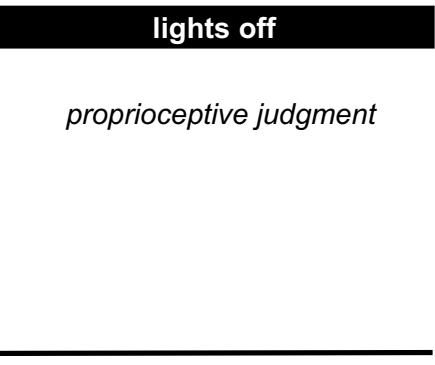

$1 \min$

Fig. 1. Experimental set-up and protocol. (A) Electrical stimulations were performed with a bipolar, binaural, configuration, with a pair of electrodes located at the mastoid processes (galvanic vestibular stimulation, GVS) or at the level of the neck (sham stimulation). Left GVS and left sham stimulation both refer to a configuration with the anode (+) located to the left side and the cathode (-) located to the right side. Participants look at a left rubber hand through a two-way mirror (B) while their own left hand (not visible) and the rubber hand were stroked synchronously or asynchronously with two paintbrushes (C). (D) Stroking was applied during 1 min while an electrical stimulation (rectangular current) was delivered. After 1 min, the lights under the two-way mirror were turned off and the rubber hand was no longer visible. Participants had to verbally report the felt position of their left index finger (proprioceptive judgment) according to the reflection of a ruler in the mirror.

\subsection{Experimental device for the rubber hand illusion}

Experiments were performed with participants seated on a chair behind a table (Fig. 1B and C). The participant's head was restrained using a head-rest that was adjustable in height and fixated to the table. Participants wore head-phones delivering white noise to avoid any auditory interference.

We used an experimental device similar to the one described in Tsakiris and Haggard (2005). A black wooden frame $(100 \times 50 \mathrm{~cm})$ was placed on the table $\sim 10 \mathrm{~cm}$ in front of the participant's trunk. The wooden frame was covered by a two-way mirror $23 \mathrm{~cm}$ above the table. A black paper sheet was placed under the left third and under the right third of the mirror in order to hide the participant's left and right hand from the participant's point of view. At the center of the woo- 
den frame we placed a realistic left rubber hand that was aligned with the participant's body midsagittal plane. The rationale to investigate representation of the left hand was based on clinical observations that somatoparaphrenia usually affects the patients' left hand (Vallar \& Ronchi, 2009). The rubber hand was located at $24.5 \mathrm{~cm}$ from the participant's left hand (distance between the middle of the index fingers). The proximal parts of the participant's hands and of the left rubber hand were covered by a black fabric.

The two-way mirror enabled us to show the left rubber hand in front of the participants during the tactile stimulations, when the lights were turned on in the wooden frame (Fig. 1D, left part). The rubber hand was hidden during the proprioceptive judgment by turning off the lights in the wooden frame (Fig. 1D, right part). To perform the proprioceptive judgment, we used a ruler placed $\sim 20 \mathrm{~cm}$ above the mirror on a metallic frame. As the lights were turned off during the proprioceptive judgment, participants were able to see the reflection of the ruler on the mirror and to localize their left index finger with respect to this ruler (Tsakiris \& Haggard, 2005).

\subsection{Experimental procedures for the rubber hand illusion}

\subsubsection{General procedure}

Before each session, the experimenter placed the participant's left hand at a fixed position on the table and the lights were turned on in the wooden frame so that the left rubber hand was visible from the participants. The experimenter thus stroked both the participants' left hand and the left rubber hand with two identical paintbrushes, either synchronously or asynchronously, during $1 \mathrm{~min}$ (Fig. 1C). Although previous studies have used longer durations of stroking (Tsakiris \& Haggard, 2005), the illusion develops on average within $15 \mathrm{~s}$ after the onset of tactile stroking (Lloyd, 2007). Here, a 1-min stroking was chosen to ascertain a safe and bearable electrical stimulation. During this 1-min stroking period, the participants also received either left or right GVS, left or right sham stimulation, or no electrical stimulation. The electrical stimulation consisted of a rectangular current at the individual amplitude determined prior to the experiment. The order in which these five conditions were presented was randomized across participants.

\subsubsection{Proprioceptive judgment}

After the 1-min stroking, the left rubber hand was hidden by turning off the lights in the wooden frame, and the participants saw the reflection of the ruler in the two-way mirror. They were asked to indicate the perceived position of their left index finger as spontaneously as possible by verbally reporting the precise number on the ruler corresponding to the position of their index finger, as if they projected a vertical line from the tip of their index finger to the ruler. In order to avoid participants repeating the same value for each trial, the ruler was always presented with a different offset. After each session, the participants' left hand was passively moved back and forth before the next condition started.

\subsubsection{Subjective report}

After each experimental condition participants filled-out a 12-item questionnaire (Table 1) adapted from the 9-item questionnaire from Botvinick and Cohen (1998), as well as from the items questionnaires \#20, 22 and 26 from Longo et al. (2008) to investigate "affective" components of the illusion. Participants were asked to indicate their level of agreement with each of the 12 items using a 7-point Likert scale ranging from 1 ("I totally disagree") to 7 ("I totally agree").

\subsection{Experimental procedures for the control proprioceptive judgment task}

We also tested the influence of GVS and sham stimulations on hand localization (proprioceptive judgment task) without any visuo-tactile conflict or tactile stroking (see Supplementary material, Fig. S1A). The participant's left hand was passively placed by the experimenter at a fixed position on the table. Participants were instructed to keep their eyes closed and they received either left or right GVS, left or right sham stimulation, or no electrical stimulation for 1 min. Neither the rubber hand nor the participants' left hand were stroked with the paintbrushes. Ten seconds after the stimulation onset they were instructed to open their eyes and they verbally reported the perceived position of their left index finger according to the reflection of the ruler in the two-way mirror. Immediately after that, they were instructed to close their eyes again and the experimenter passively moved the participant's hand and put it back to the initial position. In total, this procedure was repeated four times during the 1-min galvanic stimulation. This procedure was used in order to measure how seeing the rubber hand (without tactile stroking) can affect the localization of the participant's left hand during the different types of stimulation. After the offset of the galvanic stimulation, participants were asked a last time to indicate the perceived position of their left index finger (this last measure is comparable to the measure after the 1 min of stroking during the experimental condition). Again, the order in which the conditions were presented was randomized across participants.

\subsection{Questionnaires on illusory movement and side effects of GVS}

After the period when the current amplitudes for galvanic stimulations were determined, a $10 \mathrm{~s}$ rectangular electrical stimulation was delivered to the participants who were instructed to focus on their subjective experience during the stimulation. Immediately after, participants were given a questionnaire designed to evaluate the subjective sensations during right and left GVS and sham stimulations. They reported the magnitude of the sensation of rotation/translation of the body 


\section{Table 1}

The 12 questions used to assess subjective experiences during the rubber hand illusion. Mean subjective ratings ( \pm standard error to the mean) are reported for each question and for synchronous and asynchronous tactile stroking. $F$ and $P$ statistics for the main effect of Synchrony (synchronous versus asynchronous stroking) are reported for each question. Questions written with bold characters show a significant main effect of Synchrony.

\begin{tabular}{|c|c|c|c|}
\hline Questions & $\begin{array}{l}\text { Synchronous stroking } \\
\text { (subjective rating) }\end{array}$ & $\begin{array}{l}\text { Asynchronous stroking } \\
\text { (subjective rating) }\end{array}$ & F-statistics \\
\hline $\begin{array}{l}\text { Q1. It seemed as if I were feeling the touch of the paintbrush in the } \\
\text { location where I saw the rubber hand touched }\end{array}$ & $5.2 \pm 0.2$ & $1.8 \pm 0.1$ & $\begin{array}{l}F_{(1,15)}=58.99 \\
P<.00001\end{array}$ \\
\hline $\begin{array}{l}\text { Q2. It seemed as though the touch I felt was caused by the paintbrush } \\
\text { touching the rubber hand }\end{array}$ & $4.9 \pm 0.2$ & $1.9 \pm 0.1$ & $\begin{array}{l}F_{(1,15)}=67.84 \\
P<.00001\end{array}$ \\
\hline Q3. I felt as if the rubber hand were my hand & $4.6 \pm 0.2$ & $2.2 \pm 0.2$ & $\begin{array}{l}F_{(1,15)}=37.75 \\
P<.00001\end{array}$ \\
\hline $\begin{array}{l}\text { Q4. It felt as if my (real) hand were drifting towards the right (towards } \\
\text { the rubber hand) }\end{array}$ & $2.7 \pm 0.2$ & $2.1 \pm 0.2$ & $\begin{array}{l}F_{(1,15)}=7.90 \\
P=.01\end{array}$ \\
\hline Q5. It seemed as if I might have more than one left hand or arm & $2.4 \pm 0.2$ & $2.5 \pm 0.2$ & $\begin{array}{l}F_{(1,15)}=0.13 \\
P=.73\end{array}$ \\
\hline $\begin{array}{l}\text { Q6. It seemed as if the touch I was feeling came from somewhere between } \\
\text { my own hand and the rubber hand }\end{array}$ & $2.5 \pm 0.2$ & $2.4 \pm 0.2$ & $\begin{array}{l}F_{(1,15)}=0.01 \\
P=.92\end{array}$ \\
\hline Q7. It felt as if my (real) hand were turning 'rubbery' & $3.4 \pm 0.2$ & $2.4 \pm 0.2$ & $\begin{array}{l}F_{(1,15)}=9.78 \\
P=.007\end{array}$ \\
\hline $\begin{array}{l}\text { Q8. It appeared (visually) as if the rubber hand were drifting towards the } \\
\text { left (towards my hand) }\end{array}$ & $1.9 \pm 0.2$ & $1.9 \pm 0.2$ & $\begin{array}{l}F_{(1,15)}=0.30 \\
P=.59\end{array}$ \\
\hline $\begin{array}{l}\text { Q9. The rubber hand began to resemble my own (real) hand, in terms } \\
\text { of shape, skin tone, freckles or some other visual feature }\end{array}$ & $4.1 \pm 0.2$ & $2.8 \pm 0.2$ & $\begin{array}{l}F_{(1,15)}=11.07 \\
P=.005\end{array}$ \\
\hline Q10. I found the touch of the paintbrush on my hand was pleasant & $5.0 \pm 0.2$ & $4.1 \pm 0.2$ & $\begin{array}{l}F_{(1,15)}=10.45 \\
P=.006\end{array}$ \\
\hline Q11. I found myself liking the rubber hand & $4.1 \pm 0.2$ & $3.0 \pm 0.2$ & $\begin{array}{l}F_{(1,15)}=9.21 \\
P=.008\end{array}$ \\
\hline Q12. I found that experience enjoyable & $4.5 \pm 0.2$ & $3.7 \pm 0.2$ & $\begin{array}{l}F_{(1,15)}=8.40 \\
P=.01\end{array}$ \\
\hline
\end{tabular}

$P<.05$.

and visual field in the roll, yaw and pitch planes using a 7-point scale ranging from 0 ("no motion") to 6 ("strong motion"). Finally, at the end of the experiment, we asked participants about the strength of the experienced side effects during GVS (after Lenggenhager et al., 2008, modified after the Simulator Sickness Questionnaire of Lane \& Kennedy, 1988).

\section{Results}

\subsection{Subjective reports during the rubber hand illusion}

The strength of the rubber hand illusion was analyzed with a repeated-measures ANOVA ran on the subjective rating with the within-subjects factors Synchrony (synchronous, asynchronous stroking), Stimulation (GVS left, GVS right, sham left, sham right, without stimulation) and Question (Q1-Q12). This analysis revealed a significant main effect of Synchrony $\left(F_{(1,15)}=36.13 ; P<.0001\right)$ and Question $\left(F_{(11,165)}=9.85 ; P<.0001\right)$. In addition there was a significant interaction of Synchrony $\times$ Question $\left(F_{(11,165)}=17.60 ; P<.00001\right)$ as well as an interaction of Stimulation $\times$ Question $\left(F_{(44,660)}=2.09\right.$; $P<.0001)$. These interactions are described below.

Table 1 shows the data for the interaction of Synchrony $\times$ Question. Post-hoc analyzes (planned comparisons) confirmed that the illusion was stronger after synchronous stroking than after asynchronous stroking for items Q1-Q4, Q7 and Q9 (all $P<.01$ ). As predicted and as classically reported, the rating in the synchronous condition was particularly high for the first three items (Botvinick \& Cohen, 1998), which have been taken as indicative of the rubber hand illusion, including the feeling of ownership (e.g. "I felt as if the rubber hand were my hand") and illusory touch (e.g. "It seemed as if I were feeling the touch of the paintbrush in the location where I saw the rubber hand touched"). Regarding the three items related to affective aspects (Q10-Q12; e.g. "I found the touch of the paintbrush on my hand was pleasant"; "I found myself liking the rubber hand"), participants gave significantly lower ratings after asynchronous stroking than after synchronous stroking (all $P<.05$ ), indicating that asynchronous stroking was more likely associated with unpleasantness.

Fig. 2 illustrates the interaction of Stimulation $\times$ Question for the first three items best describing the illusion.

\subsubsection{Touch}

Post-hoc analyzes indicated that left GVS significantly increased the subjective rating for item Q2 ("It seemed as though the touch I felt was caused by the paintbrush touching the rubber hand") with respect to the condition without stimulation $\left(F_{(1,15)}=6.92 ; P<.05\right)$, as well as with respect to right sham stimulation $\left(F_{(1,15)}=6.13 ; P<.05\right)$. By contrast, there was no significant influence of right GVS on the subjective rating $\left(F_{(1,15)}=2.07 ; P=.17\right)$. Statistical analyzes did not reveal any influence of left GVS $\left(F_{(1,15)}=2.87 ; P=.11\right)$ or right GVS $\left(F_{(1,15)}=.94 ; P=.35\right)$ on the subjective rating for item Q1 ("It seemed as if I were feeling the touch of the paintbrush in the location where I saw the rubber hand touched"). 
Q1 - It seemed as if I were feeling the touch of the paintbrush in the location where I saw the rubber hand

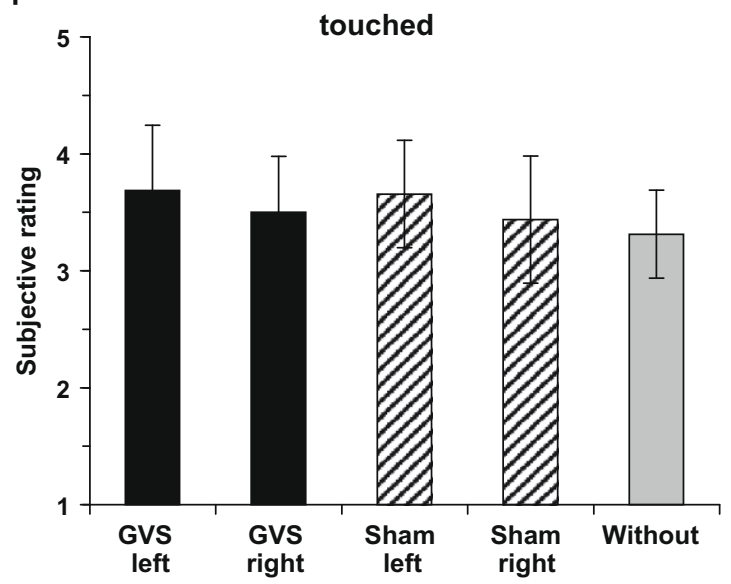

Q2 - It seemed as though the touch I felt was caused by the paintbrush touching the rubber hand

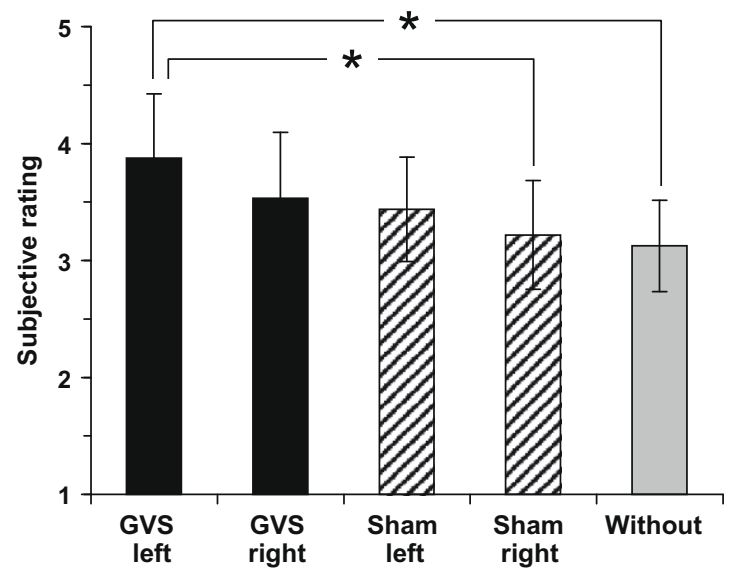

Q3 - I felt as if the rubber hand were my hand

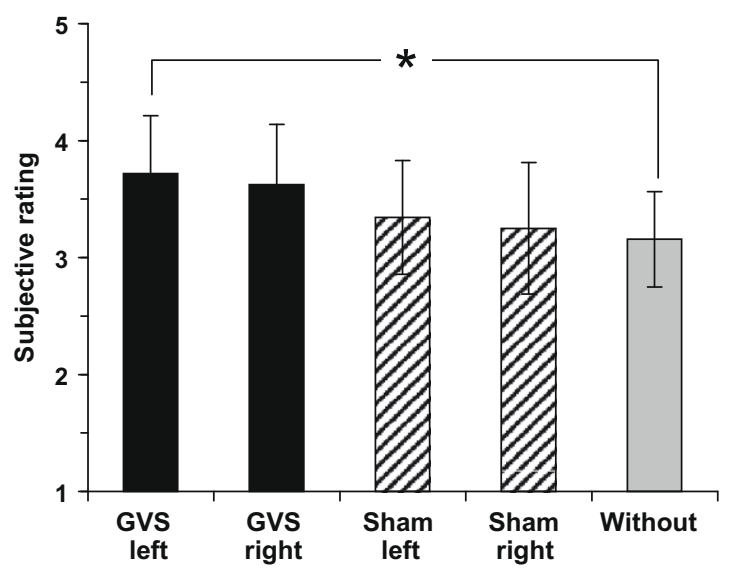

Fig. 2. Mean subjective ratings for items Q1-Q3, regarding illusory touch and ownership of the rubber hand. Vertical bars represent standard error to the mean. *Statistically significant difference, $P<.05$. 


\subsubsection{Ownership}

There was an additional influence of left GVS on ownership of the rubber hand as indicated by the subjective ratings for item Q3 ("I felt as if the rubber hand were my hand"). Participants rated item Q3 significantly higher for left GVS than for the condition without stimulation $\left(F_{(1,15)}=6.11 ; P<.05\right)$. No influence of right GVS was shown for item Q3 $\left(F_{(1,15)}=2.97 ; P=.11\right)$.

\subsubsection{Affect}

Fig. 3 illustrates how the type of stimulation influences affective components of the experience. The type of stimulation did not influence the emotional affinity towards the rubber hand (Q11: "I found myself liking the rubber hand"), but did influence the pleasantness of the experience (Q12: "I found that experience enjoyable"). Right and left GVS and sham stimulation were associated with significantly lower ratings than the condition without stimulation, meaning less pleasant experience.

\subsection{Proprioceptive drift during the rubber hand illusion}

The proprioceptive drift reflecting errors in hand localization was calculated as the difference between the position where the participants localized their left index finger while directly viewing it (uncovered) and the position of the index finger verbally reported after the 1 min tactile stroking (covered). Positive values refer to a proprioceptive drift towards the rubber hand (Fig. 4). A repeated-measures ANOVA ran on the proprioceptive drift with the within-subjects factors Synchrony and Stimulation revealed a main effect of Synchrony $\left(F_{(1,15)}=19.31 ; P<.001\right)$. This main effect of Synchrony indicated a larger proprioceptive drift after the 1 min of synchronous stroking (mean \pm SEM: $1.9 \pm 0.4 \mathrm{~cm}$ ) as compared to the asynchronous stroking $(0.5 \pm 0.4 \mathrm{~cm})$. There was no significant effect of the Stimulation $\left(F_{(4,60)}=0.33 ; P=.85\right)$ and no significant interaction of Synchrony $\times$ Stimulation $\left(F_{(4,60)}=0.40 ; P=.80\right)$.

Visual inspection of the data shows a non-significant trend (Fig. 4) for higher proprioceptive drift after left GVS than when no stimulation was applied. Data plotted in Fig. 5 illustrate the relations between the average proprioceptive drift towards the rubber hand and the average subjective rating for each of the five stimulation conditions (after synchronous stroking). The data obtained for Q2 and Q3 indicate that the proprioceptive drift correlated positively with the subjective ratings and that that left GVS was associated with the largest values of proprioceptive drift and the highest rating scores. Detailed analyzes of the correlations indicated that this relation was significant for $\mathrm{Q} 2\left(r^{2}=.94 ; P=.006\right.$; Fig. 5B $)$ and nearly significant for Q3 $\left(r^{2}=.73 ; P=.06\right.$; Fig. 5C). The coefficient of correlation was very low for Q1 (for which there was no influence of left GVS) and no correlation between proprioceptive drift and the subjective rating was found $\left(r^{2}=.02 ; P=.83\right.$; Fig. 5A). Analyzing the correlations for a control questionnaire item that was not associated with the illusion also indicated no correlation between the subjective rating and the proprioceptive drift (e.g. Q11: "I found myself liking the rubber hand": $r^{2}=.21 ; P=.44$ ), suggesting a rather specific influence of left GVS on Q2 and Q3.

\subsection{Hand localization, baseline conditions}

The influence of the type of stimulation on hand localization (without tactile stroking) was assessed once immediately after a $1 \mathrm{~min}$ period of galvanic stimulation, as well as four times during this period (Supplementary material, Fig. S1). A repeated-measures ANOVA ran on the performance in hand localization after the offset of the 1-min galvanic stimulation indicated no significant influence of the Stimulation $\left(F_{(5,11)}=1.28 ; P=.34\right)$ (Supplementary material, Fig. S1B). The errors were

Q11 - I found myself liking the rubber hand

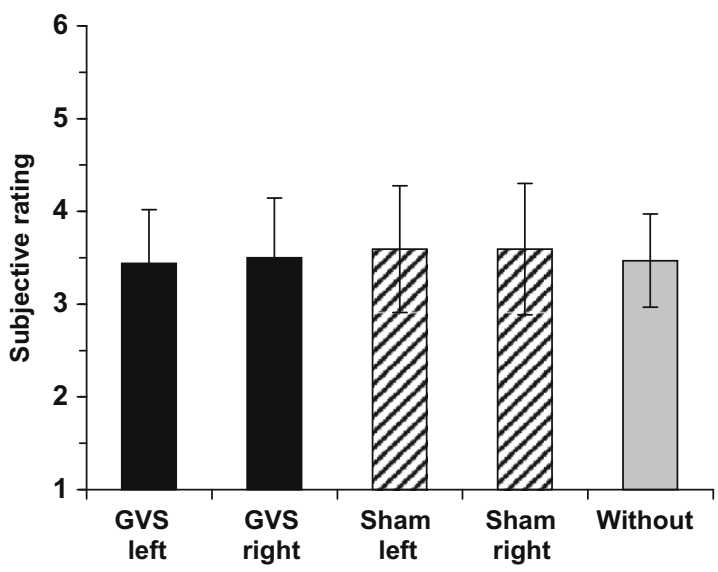

\section{Q12 - I found that experience enjoyable}

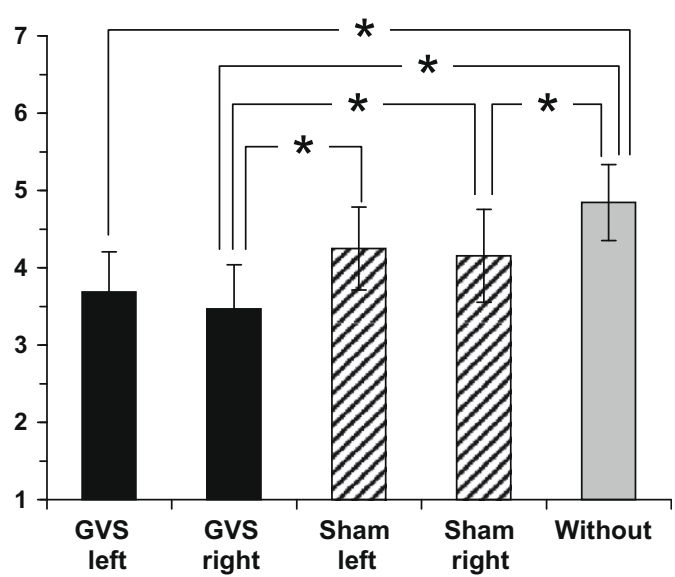

Fig. 3. Mean subjective ratings for affective aspects (Q11) and pleasantness (Q12) of the experience. Vertical bars represent standard error to the mean. ${ }^{*}$ Statistically significant difference, $P<.05$. 


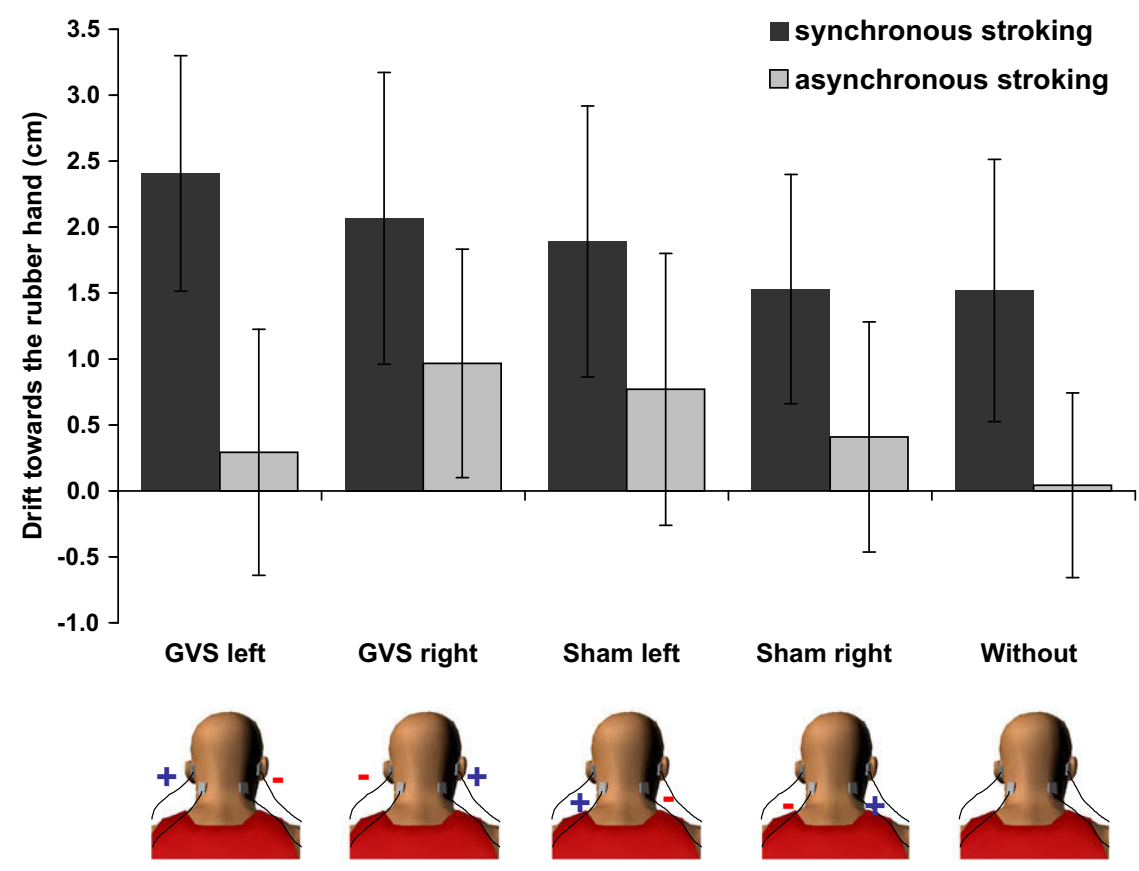

Fig. 4. Mean proprioceptive drift towards the rubber hand measured after synchronous (dark gray histograms) and asynchronous (light gray histograms) stroking. Zero represents the real position of the participant's left index finger. Vertical bars represent standard error to the mean.

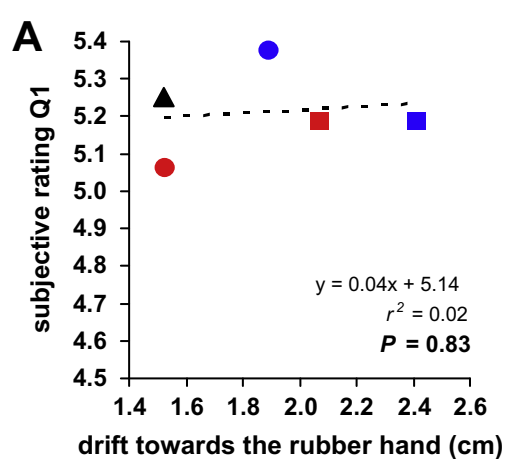

drift towards the rubber hand $(\mathrm{cm})$
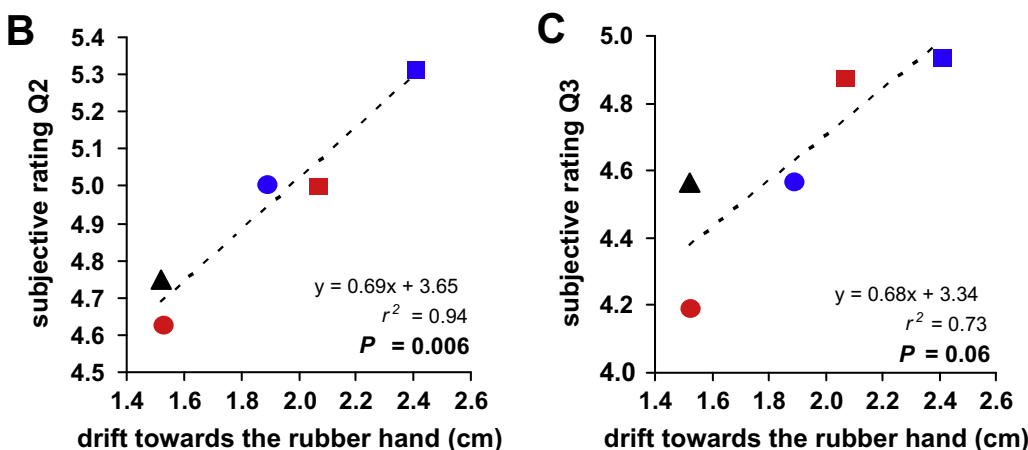

drift towards the rubber hand $(\mathrm{cm})$
GVS left

sham left

\section{GVS right}

\section{sham right}

Fig. 5. Correlations between the subjective ratings for questionnaire items Q1-Q3 and the proprioceptive drift measured after synchronous stroking in the condition without galvanic stimulation. Each symbol represents the average data for an experimental condition (GVS: squares; sham stimulation: circles; without: triangle). Positive values on the $x$ axis indicate drifts towards the rubber hand.

very small and $t$-tests (one-tailed paired $t$-tests) performed with respect to zero revealed no significant deviation with respect to this value. For the four performances recorded during the 1-min galvanic stimulation the mean of the four consecutive judgments per participant was entered into an ANOVA (Supplementary material, Fig. S1C). Statistical analysis revealed no effect of the Stimulation $\left(F_{(5,11)}=0.84 ; P=.55\right)$. Individual $t$-tests performed to compare the accuracy of hand localization with respect to zero revealed no significant deviation with respect to this value.

\subsection{Additional questionnaires}

\subsubsection{Illusory motion}

All participants reported illusory motion of the body and/or of the visual field during GVS (Supplementary material, Fig. S2). Most participants reported an illusory sensation of body rotation either in the roll plane (GVS left: $75 \%$ of the 
participants, GVS right: $88 \%$ ), pitch plane (GVS left: $44 \%$, GVS right: $25 \%$ ), or yaw plane (GVS left: $38 \%$, GVS right: $31 \%$ ). Some participants also reported an illusory sensation of body translation along the mediolateral axis (GVS left: 50\%, GVS right: 56\%), anteroposterior axis (GVS left: 38\%, GVS right: 38\%) or vertical axis (GVS left: 13\%, GVS right: 13\%). Furthermore, most participants experienced an illusory motion of the visual field (GVS left: 63\%, GVS right: 63\%). The higher subjective ratings were for perceived body rotation in the roll plane (Supplementary material, Fig. S2A) and body translation along the mediolateral axis (Supplementary material, Fig. S2B). Statistical analyzes revealed that left GVS and right GVS evoke illusory motion of similar amplitude for body rotations $\left(F_{1,15}=0.04 ; P=.85\right)$ as well as for body translations $\left(F_{1,15}=0.01 ; P=.91\right)$.

\subsubsection{Other effects induced by GVS}

Most participants reported mild to moderate pain $(81 \%)$ or heat sensation $(75 \%)$ on the skin under the electrodes (Supplementary material, Fig. S3). Other side effects reported were general discomfort (75\%), vertigo (63\%) and nausea (63\%).

\section{Discussion}

The present study was designed to investigate whether vestibular signals may influence illusory touch, hand ownership and hand localization during the rubber hand illusion. We found that left anodal galvanic vestibular stimulation (GVS) increases illusory touch and ownership for the rubber hand as compared to the condition without any stimulation.

\subsection{Subjective reports}

The present data suggest that GVS influences subjective aspects of bodily self-consciousness in healthy subjects. We found an influence of left anodal GVS on ownership of the rubber hand. Thus, participants scored higher on item Q3 ("I felt as if the rubber hand were my hand") after left GVS than when no galvanic stimulation was applied. Additionally, we found an influence of left GVS on illusory touch as participants estimated the stroking felt on their left hand to be more likely caused by the stroking of the rubber hand they were looking at as compared to when no galvanic stimulation was applied (Q2: "It seemed as though the touch I felt was caused by the paintbrush touching the rubber hand"). These two questions have been described to be the most relevant for the rubber hand illusion (Botvinick \& Cohen, 1998; Schutz-Bosbach et al., 2009; Slater, Perez-Marcos, Ehrsson, \& Sanchez-Vives, 2008) and our data thus suggest that left GVS may potentiate both classical phenomenological aspects of the rubber hand illusion.

\subsubsection{Functional mechanisms}

As shown in the present study and in previous work (Botvinick \& Cohen, 1998; Kammers, de Vignemont et al., 2009; Longo et al., 2008; Pavani et al., 2000), participants tend to merge the stroking felt on their left hand with the stroking seen on the rubber hand in a single event: the tactile stroking felt by the participants was perceived as if it were evoked by the paintbrush touching the rubber hand. This observation points to the fact that in this specific experimental condition vision may dominate for body part representation, a phenomenon that has been referred to in the literature as "visual capture" (Pavani et al., 2000).

How may vestibular stimulation alter visual capture? Because our data suggest that both the illusory feeling of touch and ownership are potentiated by left GVS, we argue that GVS may have interfered with the integration of multisensory signals from the participant's left hand, enhancing visual capture of tactile signals (from the rubber hand) and illusory ownership. We hypothesize that three non-exclusive mechanisms may account for this. GVS may have interfered with visual capture of tactile signals by visual mechanisms, by tactile mechanisms, and/or directly by visuo-tactile mechanisms. Visuo-tactile integration was controlled by manipulating the synchrony of the tactile and visual "touches". The fact that both illusory touch and ownership were increased irrespective of the synchrony of the stroking suggests that GVS increased visual capture (or increased reliance on visual signals as compared to tactile signals) not by directly interfering with visuo-tactile integration, but by interfering with visual (and/or tactile) mechanisms. Bodily perception constantly depends on weighting of vestibular, visual and somatosensory signals (Ernst \& Banks, 2002) and the present experimental manipulation of vestibular signals may have modified the respective weighting of visual and tactile signals. There is evidence to suggest that changes in vestibular signals usually increase the weighting of visual cues. First, the dependence on visual cues (or 'visual field dependence') typically increases in humans exposed to microgravity, who relied more on visual cues for self-orientation and perception of the vertical (Young, Oman, Watt, Money, \& Lichtenberg, 1984). Second, fluctuating vestibular function, as observed in patients with abnormal peripheral vestibular signals, also increases visual field dependence and these patients are more sensitive to various experimental manipulations of the visual surrounding (Bronstein, Yardley, Moore, \& Cleeves, 1996; Lopez, Lacour, Ahmadi, Magnan, \& Borel, 2007; Lopez, Lacour, Magnan, \& Borel, 2006). Thus, we propose that an increase in visual capture (and stronger illusory ownership and touch) after left GVS could result from our participants' enhanced reliance on the seen stroking of the rubber hand irrespective of the synchrony of the visuo-tactile stroking. And such a general increase of visual capture could be based on the fact that there is always a low level of ownership for the rubber hand even without synchronous stroking (e.g. Hägni et al., 2008). Although questionnaire ratings were low in the asynchronous condition for Q2 and Q3, it is possible that left GVS may have increased the weak baseline level of ownership and illusory touch in this condition. Recent literature on the rubber hand illusion suggests that visual capture is a strong mechanism because a rubber or virtual 
hand presented in an anatomically correct position might be incorporated even without tactile stroking (Hägni et al., 2008; Pavani et al., 2000). Furthermore, the asynchronous stroking condition, which is classically considered as a control condition, may also be associated with illusory sensations such as a feeling of deafferentation (Longo et al., 2008). GVS could thus have rendered less clear the distinction between self and non-self objects and this even in the condition where the illusory selfattribution of the rubber hand is normally weaker (asynchronous stroking).

GVS may also have increased visual capture by interfering with tactile mechanisms leading to a decreased reliance on tactile signals. This is in line with clinical observations that the perceived shape and size of the body and body parts might be modified in patients suffering from acute peripheral vestibular disorders (Bonnier, 1905; Yen Pik Sang et al., 2006) and that such modifications might also be evoked during artificial stimulations of the vestibular receptors (André et al., 2001; Le Chapelain et al., 2001; Yen Pik Sang et al., 2006). An interfering effect of vestibular stimulation with tactile mechanisms is further supported by neurons responding to both vestibular stimulation and tactile stimulation applied to the limbs. Such neurons have been described in the parieto-insular vestibular cortex (Grüsser, Pause, \& Schreiter, 1990), the ventral intraparietal area (Bremmer, Klam, Duhamel, Ben Hamed, \& Graf, 2002), and the primary somatosensory cortex (Schwarz \& Fredrickson, 1971). Therefore, by interfering with tactile signals from one's own body parts, GVS may have decreased the importance of such signals, potentiating the illusory incorporation of a fake hand into the body representation. So far these explanations are rather speculative and further work is needed to clarify the specific effects of GVS on visual, tactile, and visuo-tactile mechanisms of bodily self-consciousness.

As the visuo-tactile stroking during the rubber hand illusion relies on spatial as well as temporal cues, the above changes related to GVS may be due to the influence of GVS on the timing of visual and tactile signals. Thus, the temporal matching between the stroking felt on the real hand and the stroking seen on the rubber hand may have been altered by GVS and this could have influenced ratings for illusory touch and ownership. There is some evidence that natural vestibular stimulations may affect the perception of temporal intervals (Capelli, Deborne, \& Israel, 2007) as well as the perceived timing between tactile stimuli applied simultaneously or not to the right hand and left hand (Figliozzi, Guariglia, Silvetti, Siegler, \& Doricchi, 2005). Therefore, GVS may have influenced the rubber hand illusion by interfering with the perceived timing of the seen and felt stroking. This could be an additional reason why the influence of left GVS on subjective reports was found irrespective of the synchrony of the visuo-tactile stroking, thus modifying also subjective reports in the case of asynchronous stroking between the real hand and the rubber hand.

In conclusion, these questionnaire data indicate that GVS may have modified visual and/or tactile signals prior to visualtactile integration through interference with spatial and/or temporal mechanisms of intersensory matching, leading to an increase in the strength of illusory touch and ownership of the rubber hand. This idea is supported by clinical evidence that misattribution, mislocalization or duplication of the body or body parts may be associated with vestibular disturbances from central and peripheral origin (Blanke et al., 2002; Bonnier, 1905; Hécaen \& de Ajuriaguerra, 1952; Lopez et al., 2008).

\subsubsection{Differential influence of left versus right anodal GVS?}

Our data suggest a selective influence of left anodal GVS on body ownership as well as illusory touch, and only compared to the condition without any galvanic stimulation. Left anodal-right cathodal GVS is known to decrease the firing rate of the vestibular nerve on the left side and to increase it on the right side (Fitzpatrick \& Day, 2004; Goldberg et al., 1984). This stimulation mimics an inhibition of the left ear and an activation of the right ear. We suggest that the influence of left anodal GVS on ownership of the rubber hand and illusory touch was related to the capacity of this artificial stimulation to interact with the neural mechanisms of body part ownership, predominantly in the right hemisphere representing the tested left hand. This is supported by the effects of unilateral caloric vestibular stimulation in brain-damaged patients that have motivated the present study: caloric vestibular stimulation was shown to transiently reduce deficits of body part ownership in patients suffering from left hand somatoparaphrenia or to restore awareness for tactile stimuli (Bisiach et al., 1991; Bottini et al., 1995; Rode et al., 1992). In a case report by Bisiach and colleagues (1991), the patient's left arm was temporarily experienced as the patient's own arm after irrigation of the left (contralesional) ear with cold water. In our study, we showed that after left anodal GVS (associated with decreasing the firing of vestibular neurons in the left ear) a fake hand was more strongly selfattributed and touches applied to the fake hand were felt as if applied to one's own hand. These two reports are thus comparable with respect to the laterality of the vestibular stimulation and its effects on body ownership: Bisiach et al. (1991) were able to reduce disownership for the patient's left hand using an inhibition of the left, contralesional, vestibular apparatus with cold water, and we were able to increase ownership of the fake hand using left, anodal, GVS. Altogether, these data suggests that the laterality of experimental vestibular stimulation matters, compatible with observations suggesting that bilateral caloric vestibular stimulations are ineffective in modifying spatial deficits (Rode et al., 2002). It should also be noted that the side-specific effects reported here were not due to different current amplitudes for left and right GVS (see Methods). One can finally wonder whether the lateralized effects reported in our study (influence of left, but not right, GVS) could not be the consequence of only testing the participant's left hand. Since we did not test the participant's right hand we cannot exclude that the opposite pattern would have been observed if the right hand had been tested, or if the left cerebral hemisphere is generally less involved in body part representation and attribution. Yet, abnormal ownership of the right hand is extremely rare in brain-damaged patients (Vallar \& Ronchi, 2009), suggesting a right cerebral dominance for body part ownership. Nevertheless, that the activation of the left labyrinth is also effective in interfering with ownership for right limbs is suggested by a clinical observation. In a rare clinical case of disownership of the right hand, after a left cerebral stroke, right cold caloric vestibular stimulation temporarily alleviated symptoms, while left cold caloric stimulation was ineffective 
(Schiff \& Pulver, 1999). Further studies should thus be conducted in order to investigate the influence of left/right GVS on ownership for both hands.

\subsubsection{Additional factors}

In addition to its vestibular effects, GVS provided a cutaneous stimulation evoking a local tactile sensation perceived as unpleasant or painful. Might this unpleasant tactile sensation have acted as a distractor for the participants, e.g. by catching their attention to one side and thus influencing the illusion in this way? We believe that distracting effects cannot account for our results. First, sham stimulation were also judged as significantly unpleasant but were associated neither with significant changes in ownership nor illusory touch. Second, the effect on ownership of the rubber hand was only observed for left GVS, although left and right GVS were judged as equally less unpleasant and we used similar current amplitudes for left and right GVS. Third, the accuracy of hand localization recorded after GVS (both after the rubber hand illusion and during the baseline condition) did not differ from the condition without stimulation. Fourth, there is evidence in the literature that unilateral caloric vestibular stimulation (irrigation of the left ear with iced water) did not affect covert visual attention in healthy participants (Rorden, Karnath, \& Driver, 2001). Instead of speaking in favor of a distracting effect of the GVS, our data rather suggest a vestibular influence on the rubber hand illusion that well survives the unpleasantness of tactile stimulations during GVS. This supports recent behavioral data showing that painful stimuli did not influence the nature and intensity of the rubber hand illusion (Capelari, Uribe, \& Brasil-Neto, 2009). Apart from the cutaneous side effects of GVS, illusory self-motion evoked by GVS could have acted as another confounding factor. However, the subjective rating of the intensity of the perceived illusory motion did not differ between left and right GVS, and our data reveal an influence of only left GVS on the illusory ownership of the rubber hand.

These data speak against general attentional effects. Yet, our observation that left GVS influences ownership and illusory touch only if compared to the condition without any stimulation, but not as compared to left sham stimulation, suggests a weak and non-significant influence of sham stimulation. This indicates that additional factors such as cutaneous sensations, unpleasantness and pain may also have had an effect in our protocol. Some authors have speculated that potential effects of transcutaneous electrical stimulation may be due to a non-specific sensory activation orienting general attention to the stimulated side and/or to a non-specific activation of the contralateral cerebral hemisphere (Pérennou, Amblard, Leblond, \& Pélissier, 1998; Vallar et al., 1995). Others have found that transcutaneous electrical stimulation to the neck had little or no influence on spatial perception (Karnath, 1995). The present data reinforces the importance of integrating sham electrical stimulations to experimental protocols using GVS. This has not been the general rule so far, but will be necessary for future work in order to understand how cutaneous sensations, unpleasantness, pain, and attention during GVS interfere with the observed vestibular effects.

\subsubsection{Neural mechanisms}

Neuroimaging data revealed that regions for vestibular and multisensory processing overlap with those for hand ownership and are located mainly at the temporo-parietal junction (TPJ), including the posterior insula, and the posterior parietal cortex as well as the premotor cortex (Botvinick, 2004; Ehrsson, Spence, \& Passingham, 2004; Ehrsson et al., 2005; Kammers, Verhagen et al., 2009; Tsakiris, Costantini, \& Haggard, 2008; Tsakiris, Hesse, Boy, Haggard, \& Fink, 2007).

In an fMRI experiment Fink et al. (2003) identified the cortical vestibular projections using a bipolar, binaural, GVS configuration similar to the one used in the present study. These authors showed that that left anodal GVS strongly activated the right TPJ, including the superior temporal gyrus, posterior insula and inferior parietal cortex (see Fig. 6A). This vestibular

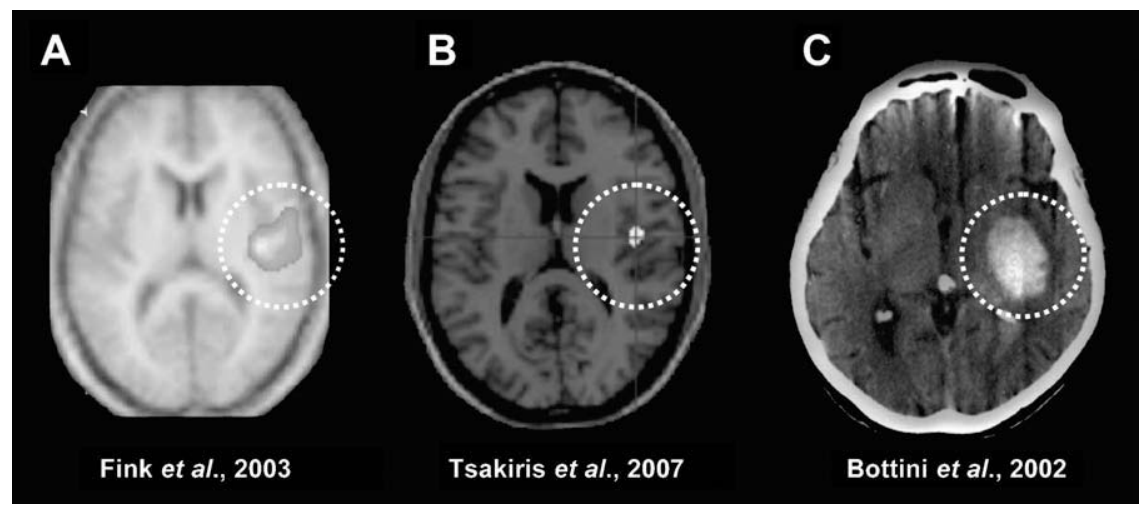

Fig. 6. Some overlapping brain regions for vestibular processing and body parts ownership. (A) Left anodal GVS/right cathodal GVS (excitation of the right and inhibition of the left vestibular apparatus) induces a significant BOLD signal increase in the right posterior insula, superior temporal gyrus and anterior inferior parietal cortex. After Fink et al. (2003). (B) In a positron emission tomography study, ownership of a fake hand (proprioceptive drift towards the rubber hand) was positively correlated to BOLD signal in the right posterior insula. After Tsakiris et al. (2007). (C) A 77 year-old right-handed woman suffering from somatoparaphrenia for her left hand (which she attributed to her niece) had a hemorrhagic lesion involving the white matter underlying the right insula, superior temporal gyrus, parietal operculum, and the precentral and postcentral gyri. After Bottini et al. (2002). 
region, centered on the posterior insula has been described as the core area of the vestibular cortex (Bense et al., 2001; Brandt \& Dieterich, 1999; Dieterich et al., 2003; Guldin \& Grüsser, 1998). An influence of vestibular signals on visual-tactile integration is also supported by electrophysiological data that described neurons responding to visual, tactile and vestibular stimulations, e.g. in the parieto-insular vestibular cortex (Grüsser et al., 1990), the ventral intraparietal area (Bremmer et al., 2002), and the somatosensory cortex (Schwarz \& Fredrickson, 1971).

Interestingly, Tsakiris et al. (2007), investigating the neural correlates of the rubber hand illusion, showed that body ownership was partly related to the activity in the posterior insula, in close proximity to the TPJ. This region thus overlapped with the core region of the vestibular cortex (see Fig. 6B). Additional evidence of the participation of the right TPJ in the sense of ownership comes from transcranial magnetic stimulation studies showing that stimulation over the right TPJ may attenuate the rubber hand illusion (Tsakiris et al., 2008).

Finally, a third line of evidence showing a relation between the right TPJ and body part ownership arises from neurological patients with abnormal sense of ownership. Somatoparaphrenia is often observed after lesion of the right temporo-parietal cortex, including the posterior insula (see Fig. 6C; Bottini et al., 2002; Cereda, Ghika, Maeder, \& Bogousslavsky, 2002; Vallar \& Ronchi, 2009). A recent analysis of lesion location in patients reporting abnormal limb ownership indicated that the right posterior insula is a crucial region for elaborating a normal sense of body part ownership (Baier \& Karnath, 2008).

Based on these data, we hypothesized that left anodal GVS interferes more likely than right GVS with the neural substrates of ownership for left body parts. The effects of left anodal GVS on the experience of ownership for the hand and illusory touch might therefore be due to interfering effects with the right TPJ and posterior insula, multimodal regions that integrate visual, tactile, proprioceptive and vestibular signals and whose integrity is required for normal sense of body part ownership, embodiment, and sense of unity between self and body (Baier \& Karnath, 2008; Blanke et al., 2002, 2004; Lenggenhager et al., 2006; Lopez et al., 2008; Tsakiris et al., 2008, 2007).

\subsection{Proprioceptive judgments}

Our data reveal a larger drift of the perceived location of the participant's left index finger towards the rubber hand after synchronous than after asynchronous stroking, as reported by many previous authors (Capelari et al., 2009; Costantini \& Haggard, 2007; Longo et al., 2008; Schutz-Bosbach et al., 2009; Tsakiris \& Haggard, 2005; Tsakiris, Prabhu, \& Haggard, 2006). This mislocalization of the participant's hand is in line with subjective reports (Q4: "It felt as if my (real) hand were drifting towards the right (towards the rubber hand)") and may reflect the visual capture by the stroking of the rubber hand (Pavani et al., 2000). Importantly however, the present data indicate that the amplitude of the proprioceptive drift was not significantly influenced by GVS. It seems therefore that there is a dissociation between the influence of GVS on subjective reports (questionnaires) and objective measurements (the proprioceptive drift). However, our correlation analysis indicates that there is a positive correlation between the influence of the experimental condition (left and right GVS, left and right sham stimulation, without) on the proprioceptive drift and on the illusory touch (Q2) and ownership (Q3). In other words, when comparing the influence of the different experimental conditions, stronger illusions are associated with larger drifts. This suggests that the effects of left GVS on the drift may have been more subtle than those on subjective reports. We also note that drift and questionnaires may not measure the same aspects of the illusion (Kammers, de Vignemont et al., 2009; Longo et al., 2008) and that actual correlations have only been found in few studies (Botvinick \& Cohen, 1998; Ehrsson et al., 2005). Furthermore, the mislocalization of the participant's hand may be resistant to external manipulation, as it was found to remain unchanged after proprioceptive feedback evoked by arm movements (Kammers, de Vignemont et al., 2009), or after tactile-painful stimuli (Capelari et al., 2009).

Finally, we found that GVS did not influence the perceived location of the participant's left hand in the different control conditions (where participants were asked to localize their left index finger without any stroking). Our data therefore do not support previously reported influence of GVS on hand localization during reaching movements (deviation towards the anodal side during GVS) (Bresciani et al., 2002; Knox et al., 2006; Mars et al., 2003). It should be noted that, in the present experimental conditions, the participant's hand was stable and palm-down on the table, whereas these previous studies (Bresciani et al., 2002; Mars et al., 2003) have mostly used actions involving either pointing to a target or reproduction of arm positions. Vestibular stimulation may thus more likely influence the perceived hand location during action, when actions involve arm and hand interactions with gravitational forces, and online readjustments of the antigravity commands to arm muscles (Lacquaniti \& Maioli, 1987).

\subsection{Conclusions}

The present results suggest an implication of vestibular signals in the conscious experiences of touch and hand ownership, thus bringing earlier observations in neurological patients with abnormal hand ownership to healthy participants under scientific scrutiny. We suggest that changes in illusory touch and hand ownership during GVS might be due to a spatial or temporal modification of visual-tactile integration, leading to an enhancement of visual capture. Such changes were selectively found for left anodal GVS and we speculate that this is due to an interference with activity in the right TPJ and posterior insula, crucial multimodal regions whose integrity is required for normal sense of body part ownership. 


\section{Acknowledgment}

This research was supported by the Swiss National Science Foundation (SINERGIA CRSII1-125135/1).

\section{Appendix A. Supplementary data}

Supplementary data associated with this article can be found, in the online version, at doi:10.1016/j.concog.2009.12.003.

\section{References}

Aglioti, S., Smania, N., Manfredi, M., \& Berlucchi, G. (1996). Disownership of left hand and objects related to it in a patient with right brain damage. NeuroReport, 8(1), 293-296.

André, J. M., Martinet, N., Paysant, J., Beis, J. M., \& Le Chapelain, L. (2001). Temporary phantom limbs evoked by vestibular caloric stimulation in amputees. Neuropsychiatry Neuropsychology Behavioral Neurology, 14(3), 190-196.

Angelaki, D. E., \& Cullen, K. E. (2008). Vestibular system: the many facets of a multimodal sense. Annual Review of Neuroscience, 31, 125-150.

Armel, K. C., \& Ramachandran, V. S. (2003). Projecting sensations to external objects: Evidence from skin conductance response. Proceedings of Biological Science, 270(1523), 1499-1506.

Aw, S. T., Haslwanter, T., Fetter, M., \& Dichgans, J. (2000). Three-dimensional spatial characteristics of caloric nystagmus. Experimental Brain Research, 134(3), 289-294.

Baier, B., \& Karnath, H. O. (2008). Tight link between our sense of limb ownership and self-awareness of actions. Stroke, 39(2), 486-488.

Bense, S., Stephan, T., Yousry, T. A., Brandt, T., \& Dieterich, M. (2001). Multisensory cortical signal increases and decreases during vestibular galvanic stimulation (fMRI). Journal of Neurophysiology, 85(2), 886-899.

Berlucchi, G., \& Aglioti, S. (1997). The body in the brain: Neural bases of corporeal awareness. Trends in Neurosciences, 20(12), 560-564.

Berlucchi, G., \& Aglioti, S. M. (2009). The body in the brain revisited. Experimental Brain Research.

Bisiach, E., Rusconi, M. L., \& Vallar, G. (1991). Remission of somatoparaphrenic delusion through vestibular stimulation. Neuropsychologia, 29(10), 1029-1031.

Blanke, O., Landis, T., Spinelli, L., \& Seeck, M. (2004). Out-of-body experience and autoscopy of neurological origin. Brain, 127(2), 243-258.

Blanke, O., \& Metzinger, T. (2009). Full-body illusions and minimal phenomenal selfhood. Trends in Cognitive Sciences, 13(1), 7-13.

Blanke, O., Ortigue, S., Landis, T., \& Seeck, M. (2002). Stimulating illusory own-body perceptions. Nature, 419(6904), 269-270.

Bonnier, P. (1905). L'Aschématie. Revue Neurologique (Paris), 12, 605-609.

Bottini, G., Bisiach, E., Sterzi, R., \& Vallar, G. (2002). Feeling touches in someone else's hand. NeuroReport, 13(2), 249-252.

Bottini, G., Paulesu, E., Sterzi, R., Warburton, E., Wise, R. J., Vallar, G., et al (1995). Modulation of conscious experience by peripheral sensory stimuli. Nature, 376(6543), 778-781.

Botvinick, M. (2004). Neuroscience. Probing the neural basis of body ownership. Science, 305(5685), 782-783.

Botvinick, M., \& Cohen, J. (1998). Rubber hands 'feel' touch that eyes see. Nature, 391(6669), 756.

Brandt, T., \& Dieterich, M. (1999). The vestibular cortex. Its locations, functions, and disorders. Annals-New York Academy of Science, 871, $293-312$.

Bremmer, F., Klam, F., Duhamel, J. R., Ben Hamed, S., \& Graf, W. (2002). Visual-vestibular interactive responses in the macaque ventral intraparietal area (VIP). European Journal of Neuroscience, 16(8), 1569-1586.

Bresciani, J. P., Blouin, J., Popov, K., Bourdin, C., Sarlegna, F., Vercher, J. L., et al (2002). Galvanic vestibular stimulation in humans produces online arm movement deviations when reaching towards memorized visual targets. Neuroscience Letter, 318(1), 34-38.

Bronstein, A. M., Yardley, L., Moore, A. P., \& Cleeves, L. (1996). Visually and posturally mediated tilt illusion in Parkinson's disease and in labyrinthine defective subjects. Neurology, 47(3), 651-656.

Capelari, E. D., Uribe, C., \& Brasil-Neto, J. P. (2009). Feeling pain in the rubber hand: Integration of visual, proprioceptive, and painful stimuli. Perception, $38(1), 92-99$.

Capelli, A., Deborne, R., \& Israel, I. (2007). Temporal intervals production during passive self-motion in darkness. Current Psychology Letters, 22(2).

Cereda, C., Ghika, J., Maeder, P., \& Bogousslavsky, J. (2002). Strokes restricted to the insular cortex. Neurology, 59(12), 1950-1955.

Costantini, M., \& Haggard, P. (2007). The rubber hand illusion: Sensitivity and reference frame for body ownership. Consciousness and Cognition, 16(2), $229-240$.

Damasio, A. R. (2000). The feeling of what happens: Body and emotion in the making of consciousness. San Diego: Harvest Books.

Day, B. L., \& Fitzpatrick, R. C. (2005). The vestibular system. Current Biology, 15(15), R583-586.

de Vignemont, F. (2007). Habeas corpus: The sense of ownership of one's own body. Mind and Language, 22(4), 427-449.

Dichgans, J., \& Brandt, T. (1978). Visual-vestibular interaction: Effects on self-motion perception and postural control. In R. Held, H. W. Leibowitz, \& H.-L. Teuber (Eds.). Handbook of sensory physiology: Perception (Vol. 8, pp. 755-804). Springer-Verlag.

Dieterich, M., Bense, S., Lutz, S., Drzezga, A., Stephan, T., Bartenstein, P., et al (2003). Dominance for vestibular cortical function in the non-dominant hemisphere. Cerebral Cortex, 13(9), 994-1007.

Ehrsson, H. H., Holmes, N. P., \& Passingham, R. E. (2005). Touching a rubber hand: Feeling of body ownership is associated with activity in multisensory brain areas. Journal of Neuroscience, 25(45), 10564-10573.

Ehrsson, H. H., Spence, C., \& Passingham, R. E. (2004). That's my hand! Activity in premotor cortex reflects feeling of ownership of a limb. Science, 305(5685), 875-877.

Ehrsson, H. H., Wiech, K., Weiskopf, N., Dolan, R. J., \& Passingham, R. E. (2007). Threatening a rubber hand that you feel is yours elicits a cortical anxiety response. Proceedings of National Academic Science USA, 104(23), 9828-9833.

Ernst, M. O., \& Banks, M. S. (2002). Humans integrate visual and haptic information in a statistically optimal fashion. Nature, 415(6870), 429-433.

Figliozzi, F., Guariglia, P., Silvetti, M., Siegler, I., \& Doricchi, F. (2005). Effects of vestibular rotatory accelerations on covert attentional orienting in vision and touch. Journal of Cognitive Neuroscience, 17(10), 1638-1651.

Fink, G. R., Marshall, J. C., Weiss, P. H., Stephan, T., Grefkes, C., Shah, N. J., et al (2003). Performing allocentric visuospatial judgments with induced distortion of the egocentric reference frame: An fMRI study with clinical implications. NeuroImage, 20(3), 1505-1517.

Fitzpatrick, R. C., \& Day, B. L. (2004). Probing the human vestibular system with galvanic stimulation. Journal of Applied Physiology, 96(6), $2301-2316$.

Fitzpatrick, R. C., Marsden, J., Lord, S. R., \& Day, B. L. (2002). Galvanic vestibular stimulation evokes sensations of body rotation. NeuroReport, 13(18), 2379-2383.

Fourneret, P., Paillard, J., Lamarre, Y., Cole, J., \& Jeannerod, M. (2002). Lack of conscious recognition of one's own actions in a haptically deafferented patient. NeuroReport, 13(4), 541-547.

Gallagher, S. (2000). Philosophical conceptions of the self: Implications for cognitive science. Trends in Cognitive Sciences, 4(1), 14-21.

Goldberg, J. M., Smith, C. E., \& Fernandez, C. (1984). Relation between discharge regularity and responses to externally applied galvanic currents in vestibular nerve afferents of the squirrel monkey. Journal of Neurophysiology, 51(6), 1236-1256.

Grüsser, O. J., Pause, M., \& Schreiter, U. (1990). Vestibular neurones in the parieto-insular cortex of monkeys (Macaca fascicularis): Visual and neck receptor responses. Journal of Physiology, 430(1), 559-583. 
Guldin, W. O., \& Grüsser, O. J. (1998). Is there a vestibular cortex? Trends in Neurosciences, 21(6), 254-259.

Hägni, K., Eng, K., Hepp-Reymond, M. C., Holper, L., Keisker, B., Siekierka, E., et al (2008). Observing virtual arms that you imagine are yours increases the galvanic skin response to an unexpected threat. PLoS ONE, 3(8), e3082.

Hécaen, H., \& de Ajuriaguerra, J. (1952). Méconnaissances et hallucinations corporelles. Intégration et désintégration de la somatognosie. Paris: Masson.

Jeannerod, M. (2006). Motor cognition: What actions tell to the self. Oxford University Press.

Kammers, M. P., de Vignemont, F., Verhagen, L., \& Dijkerman, H. C. (2009). The rubber hand illusion in action. Neuropsychologia, 47(1), 204-211.

Kammers, M. P., Verhagen, L., Dijkerman, H. C., Hogendoorn, H., De Vignemont, F., \& Schutter, D. J. (2009). Is this hand for real? Attenuation of the rubber hand illusion by transcranial magnetic stimulation over the inferior parietal lobule. Journal of Cognitive Neuroscience, 21(7), 1311-1320.

Karnath, H. O. (1995). Transcutaneous electrical stimulation and vibration of neck muscles in neglect. Experimental Brain Research, 105(2), 321-324.

Knox, J. J., Coppieters, M. W., \& Hodges, P. W. (2006). Do you know where your arm is if you think your head has moved? Experimental Brain Research, 173(1), 94-101.

Kolev, O. I. (1995). Visual hallucinations evoked by caloric vestibular stimulation in normal humans. Journal of Vestibular Research, 5(1), 19-23.

Lacquaniti, F., \& Maioli, C. (1987). Anticipatory and reflex coactivation of antagonist muscles in catching. Brain Research, 406(1-2), 373-378.

Lane, N. E., \& Kennedy, R. S. (1988). A new method for quantifying simulator sickness: Development and application of the simulator sickness questionnaire (SSQ). Orlando: Essex Corporation.

Le Chapelain, L., Beis, J. M., Paysant, J., \& Andre, J. M. (2001). Vestibular caloric stimulation evokes phantom limb illusions in patients with paraplegia. Spinal Cord, 39(2), 85-87.

Lenggenhager, B., Lopez, C., \& Blanke, O. (2008). Influence of galvanic vestibular stimulation on egocentric and object-based mental transformations. Experimental Brain Research, 184(2), 211-221.

Lenggenhager, B., Smith, S. T., \& Blanke, O. (2006). Functional and neural mechanisms of embodiment: Importance of the vestibular system and the temporal parietal junction. Reviews in the Neurosciences, 17(6), 643-657.

Lloyd, D. M. (2007). Spatial limits on referred touch to an alien limb may reflect boundaries of visuo-tactile peripersonal space surrounding the hand. Brain and Cognition, 64(1), 104-109.

Longo, M. R., Schuur, F., Kammers, M. P., Tsakiris, M., \& Haggard, P. (2008). What is embodiment? A psychometric approach. Cognition, 107(3), 978-998.

Lopez, C., \& Blanke, O. (2007). Neuropsychology and neurophysiology of self-consciousness. Multisensory and vestibular mechanisms. In A. Holderegger, B. Sitter-Liver, C. W. Hess, \& G. Rager (Eds.), Hirnforschung und Menschenbild. Beiträge zur interdisziplinären Verständigung (pp. 183-206). Fribourg, Basel: Academic Press, Schwabe.

Lopez, C., Halje, P., \& Blanke, O. (2008). Body ownership and embodiment: Vestibular and multisensory mechanisms. Neurophysiologie Clinique, 38(3), $149-161$.

Lopez, C., Lacour, M., Ahmadi, A. E., Magnan, J., \& Borel, L. (2007). Changes of visual vertical perception: A long-term sign of unilateral and bilateral vestibular loss. Neuropsychologia, 45(9), 2025-2037.

Lopez, C., Lacour, M., Magnan, J., \& Borel, L. (2006). Visual field dependence-independence before and after unilateral vestibular loss. NeuroReport, 17(8), 797-803.

Mars, F., Archambault, P. S., \& Feldman, A. G. (2003). Vestibular contribution to combined arm and trunk motion. Experimental Brain Research, 150(4), 515-519.

Mars, F., Vercher, J. L., \& Popov, K. (2005). Dissociation between subjective vertical and subjective body orientation elicited by galvanic vestibular stimulation. Brain Research Bulletin, 65(1), 77-86.

Miller, S. M., \& Ngo, T. T. (2007). Studies of caloric vestibular stimulation: Implications for cognitive neurosciences, the clinical neurosciences and neurophilosophy. Acta Neuropsychiatrica, 19, 183-203.

Moseley, G. L., Olthof, N., Venema, A., Don, S., Wijers, M., Gallace, A., et al (2008). Psychologically induced cooling of a specific body part caused by the illusory ownership of an artificial counterpart. Proceedings of National Academic Science USA, 105(35), 13169-13173.

Pavani, F., Spence, C., \& Driver, J. (2000). Visual capture of touch: Out-of-the-body experiences with rubber gloves. Psychological Science, 11(5), 353-359.

Pérennou, D. A., Amblard, B., Leblond, C., \& Pélissier, J. (1998). Biased postural vertical in humans with hemispheric cerebral lesions. Neuroscience Letters, 252(2), 75-78.

Ramachandran, V. S., \& McGeoch, P. (2007). Can vestibular caloric stimulation be used to treat apotemnophilia? Medical Hypotheses, 69(2), 250-252.

Rode, G., Charles, N., Perenin, M. T., Vighetto, A., Trillet, M., \& Aimard, G. (1992). Partial remission of hemiplegia and somatoparaphrenia through vestibular stimulation in a case of unilateral neglect. Cortex, 28(2), 203-208.

Rode, G., Tilikete, C., Luaute, J., Rossetti, Y., Vighetto, A., \& Boisson, D. (2002). Bilateral vestibular stimulation does not improve visual hemineglect. Neuropsychologia, 40(7), 1104-1106.

Rorden, C., Karnath, H. O., \& Driver, J. (2001). Do neck-proprioceptive and caloric-vestibular stimulation influence covert visual attention in normals, as they influence visual neglect? Neuropsychologia, 39(4), 364-375.

Schiff, N. D., \& Pulver, M. (1999). Does vestibular stimulation activate thalamocortical mechanisms that reintegrate impaired cortical regions? Proceedings of the Royal Society of London. Series B: Biological Sciences, 266(1417), 421-423.

Schutz-Bosbach, S., Tausche, P., \& Weiss, C. (2009). Roughness perception during the rubber hand illusion. Brain and Cognition, $20,20-21$.

Schwarz, D. W. F., \& Fredrickson, J. M. (1971). Rhesus monkey vestibular cortex: A bimodal primary projection field. Science, 172, 280-281.

Slater, M., Perez-Marcos, D., Ehrsson, H. H., \& Sanchez-Vives, M. V. (2008). Towards a digital body: The virtual arm illusion. Frontiers in Human Neuroscience, $2,6$.

Tsakiris, M., Costantini, M., \& Haggard, P. (2008). The role of the right temporo-parietal junction in maintaining a coherent sense of one's body. Neuropsychologia, 46(12), 3014-3018.

Tsakiris, M., \& Haggard, P. (2005). The rubber hand illusion revisited: Visuo-tactile integration and self-attribution. Journal of Experimental Psychology: Human Perception and Performance, 31(1), 80-91.

Tsakiris, M., Hesse, M. D., Boy, C., Haggard, P., \& Fink, G. R. (2007). Neural signatures of body ownership: A sensory network for bodily self-consciousness. Cerebral Cortex, 17(10), 2235-2244.

Tsakiris, M., Prabhu, G., \& Haggard, P. (2006). Having a body versus moving your body: How agency structures body-ownership. Consciousness and Cognition, 15(2), 423-432.

Tsakiris, M., Schutz-Bosbach, S., \& Gallagher, S. (2007). On agency and body-ownership: Phenomenological and neurocognitive reflections. Consciousness and Cognition, 16(3), 645-660.

Vallar, G., Bottini, G., Rusconi, M. L., \& Sterzi, R. (1993). Exploring somatosensory hemineglect by vestibular stimulation. Brain, 116(Pt 1), 71-86.

Vallar, G., \& Ronchi, R. (2009). Somatoparaphrenia: A body delusion. A review of the neuropsychological literature. Experimental Brain Research, 192(3), $533-551$.

Vallar, G., Rusconi, M. L., Barozzi, S., Bernardini, B., Ovadia, D., Papagno, C., et al (1995). Improvement of left visuo-spatial hemineglect by left-sided transcutaneous electrical stimulation. Neuropsychologia, 33(1), 73-82.

Vogeley, K., May, M., Ritzl, A., Falkai, P., Zilles, K., \& Fink, G. R. (2004). Neural correlates of first-person perspective as one constituent of human selfconsciousness. Journal of Cognitive Neuroscience, 16(5), 817-827.

Yen Pik Sang, F., Jauregui-Renaud, K., Green, D. A., Bronstein, A. M., \& Gresty, M. A. (2006). Depersonalisation/derealisation symptoms in vestibular disease. Journal of Neurology, Neurosurgery and Psychiatry, 77(6), 760-766.

Young, L. R., Oman, C. M., Watt, D. G., Money, K. E., \& Lichtenberg, B. K. (1984). Spatial orientation in weightlessness and readaptation to earth's gravity. Science, 225(4658), 205-208.

Zink, R., Bucher, S. F., Weiss, A., Brandt, T., \& Dieterich, M. (1998). Effects of galvanic vestibular stimulation on otolithic and semicircular canal eye movements and perceived vertical. Electroencephalography and Clinical Neurophysiology, 107(3), $200-205$. 